
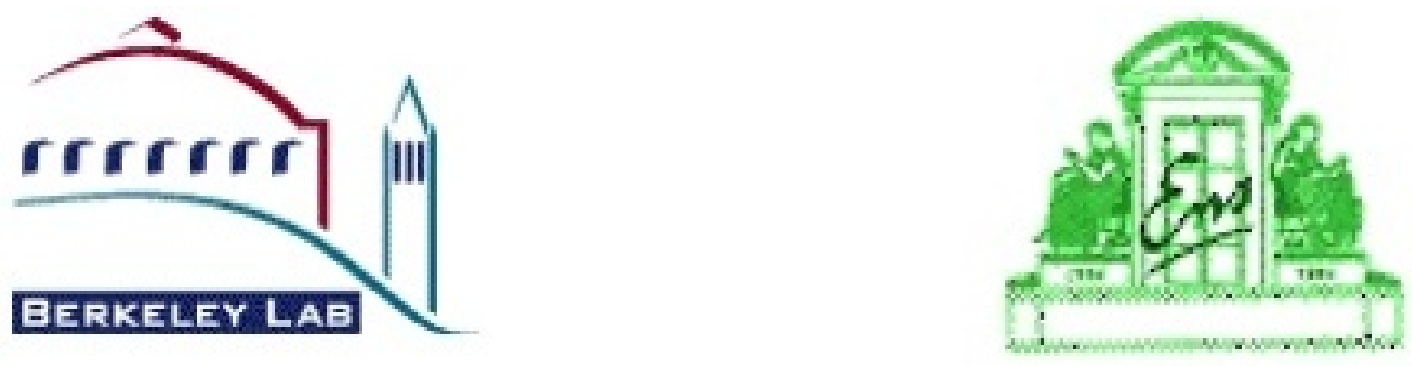

\title{
Solenoid transport of a heavy ion beam for warm dense matter studies and inertial confinement fusion
}

\author{
Julien Armijo
}

February to July 2006

Internship for 1st year of Master's at Ecole Normale Superieure, Paris, France

Advisors: Peter Seidl, William Sharp, John Barnard

Lawrence Berkeley National Laboratory, Berkeley, California, USA 


\section{Contents}

1 Magnet measurements and eddy currents in the end plates of STX 4

1.1 STX: the Solenoid Transport Experiment . . . . . . . . . . . . . . 4

1.2 Measurement of the eddy currents perturbation to the field . . . . . . . . . 5

1.3 Modeling of the phenomenon . . . . . . . . . . . . . 6

1.4 Finite-element calculation with ANSYS and importation of the realistic fields

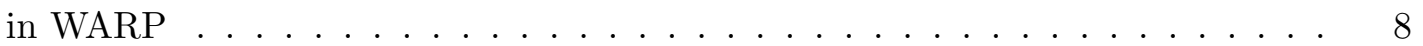

2 WARP simulations of electron leakage due to diagnostics in STX 10

2.1 Motivation: the experimental problem of the electrons leakage . . . . . . . . . 10

2.2 Method for a study using WARP . . . . . . . . . . . . . . . . . . 12

2.3 Results of the simulations . . . . . . . . . . . . . . . . . . . 12

2.4 Summary and perspectives . . . . . . . . . . . . . . . . . 14

3 Hydrodynamics of the metallic 2-phase flow in upcoming target experiment 15

3.1 The context: target experiments and hydrodynamic simulations without surface

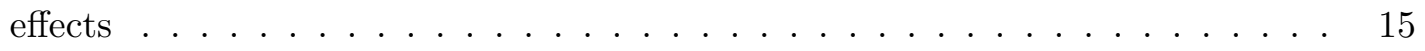

3.2 How can we describe a 2 -phase flow? . . . . . . . . . . . . . . . 17

3.3 Maximum size of droplets in an expanding flow . . . . . . . . . . . . . . . 18

3.4 Kinetic model for the evaporation of a droplet . . . . . . . . . . . . . . . . . 19 


\section{Introduction}

From February to July 2006, I have been doing research as a guest at Lawrence Berkeley National Laboratory (LBNL), in the Heavy Ion Fusion group. This internship, which counts as one semester in my master's program in France, I was very pleased to do it in a field that I consider has the beauty of fundamental physics, and at the same time the special appeal of a quest for a long-term and environmentally-respectful energy source.

What is heavy ion fusion? Nuclear reactions are undoubtedly a very powerful source of energy for human societies, with very attractive scalings. While traditional chemical energy sources, such as oil or coal, release typically $10^{7} \mathrm{~J} / \mathrm{kg}$ of fuel, fission reactions, used today in power plants, are in the range of $10^{12} \mathrm{~J} / \mathrm{kg}$, and fusion reactions, not exploited yet, release $10^{14} \mathrm{~J} / \mathrm{kg}$ of fuel.

The interest of fusion, then, is clear, especially when one considers furthermore that it should have small environmental impact (compared to chemical energy sources and even fission) and that the stock of fuel, D and T bred in the reactor, is virtually inexhaustible.

The difficulty, though, is to reach simultaneously a temperature $T \sim 10^{8} \mathrm{~K}$ and a confinement factor $\mathrm{n} \tau \sim 10^{20} \mathrm{~s} . \mathrm{m}^{-3}$. To achieve it, there is magnetic confinement fusion (MCF), and inertial confinement fusion (ICF). In ICF, the fuel is heated and compressed so fast that reactions occur before he hydrodynamic expansion has time to cool the burning material. ICF has been achieved by several means (lasers, Z-pinch, H-bombs), but heavy ion accelerators are the best promising driver, that virtually can be controlled well enough and be shot at high enough frequency ( $\sim 10 \mathrm{~Hz}$, and easily more, which seems unfeasable with lasers) to be used for an ICF power plant. That's why, at the University of California, Berkeley, several groups have been seriously working on the design of an HIF power plant [1].

One of the most attractive features in the concept of heavy ion fusion is the possibility to protect the walls of the reaction chamber from the bombarding of the $14 \mathrm{MeV}$ neutrons and the X-rays produced by the reactions. The neutrons, that are one of the biggest problems that MCF has to face, could be stopped by a thick liquid wall of molten FLiBe salt [2], which, according to its designers, could in the same time be a way to regenerate tritium from the bombarded lithium. Also, in HIF it seems easy to protect the final focusing magnets from the debris of the target. Even if there was some intrusion of debris in the solenoids, there could be an elbow-shaped turn that would not allow them to go up in the beamline. On the contrary, in laser ICF, it seems very difficult to protect the last lens from the debris.

The Heavy Ion Fusion group at LBNL Heavy ion fusion, although very promising in terms of extrapolated feasability, is being paid substantially less attention than its cousin, laser 
ICF; so that the present situation is that laser experiments have to demonstrate ignition, and in the meanwhile, real scale HIF facilities are not being built. Even though, there is plenty to do in the low energy range, and the HIF group at LBNL spreads its scientific focus on several aspects of the beam compression and transport, and is also actively preparing target experiments (planned to begin next year) that will use the HIF beam facilities to heat targets and study the properties of matter in the "Warm Dense Matter" range: densities from 0.1 to $10 \mathrm{~g} / \mathrm{cm}^{3}$ and temperatures from 0.1 to $1 \mathrm{eV}$. In the WDM range, the physics, in particular the equation of state, is not very well known [3], and its study would be very useful to simulate and understand all the phenomena that occur in or above this range (so that they transit through), in particular, all the studies in High Energy Density Physics (HEDP), some astrophysics (planet cores), and inertial fusion.

To do this research, the HIF group has two "medium scale" machines: the High Current Experiment $(\mathrm{HCX})$, an accelerator of energy $\sim 2 \mathrm{MeV} /$ ion that is mostly used to study electron cloud phenomena, and the Neutralized Drift Compression Experiment (NDCX), a $300 \mathrm{kV}$ facility that is used to shape the "best quality" beam, reaching up to 60 times longitudinal compression [4] thanks to an induction cell, and transverse compression up to mm-size [5] thanks to a neutralizing plasma that allows the beam not to feel its self space-charge.

An upcoming milestone for the group, fixed by the Department of Energy (DOE), is to simultaneously compress the NDCX beam longitudinally and transversally. Then this beam will be used for target experiments [6].

My work in the group During my stay at LBNL, I have been involved in three projects, all of them related to NDCX.

The first one, experimental and analytical, has consisted in measuring the effects of the eddy currents induced by the pulsed magnets in the conducting plates of the source and diagnostic chambers of the Solenoid Transport Experiment (STX, which is a subset of NDCX). We have modelled the effect and run finite-element simulations that have reproduced the perturbation to the field. Then, we have modified WARP, the Particle-In-Cell code used to model the whole experiment, in order to import realistic fields including the eddy current effects and some details of each magnet.

The second project has been to take part in a campaign of WARP simulations of the same experiment to understand the leakage of electrons that was observed in the experiment as a consequence to some diagnostics and the failure of the electrostatic electron trap. The simulations have shown qualitative agreement with the measured phenomena, but are still in progress.

The third project, rather theoretical, has been related to the upcoming target experiment of a thin aluminum foil heated by a beam to the $1-\mathrm{eV}$ range. At the beginning I helped by analyzing simulations of the hydrodynamic expansion and cooling of the heated material. But, progressively, my work turned into making estimates for the nature of the liquid/vapor twophase flow. In particular, I have been working on criteria and models to predict the formation of droplets, their size, and their partial or total evaporation in the expanding flow. 


\section{Chapter 1}

\section{Magnet measurements and eddy currents in the end plates of STX}

When I arrived at LBNL, one of the things that needed to be done was to measure and quantify the perturbation of the eddy currents in the end plates of STX.

\subsection{STX: the Solenoid Transport Experiment}

STX is just the name given to a particular phase of NDCX, one of the two accelerators of the HIF group. NDCX, the Neutralized Drift Compression Experiment, is a machine that has been running since 2002, and has had several interesting results, namely the transverse and the longitudinal compression of the beam [4] [5].

As shown in fig 1, STX consists of an ion source followed by a vacuum beamline made with two solenoids (there will be four in the final set-up).

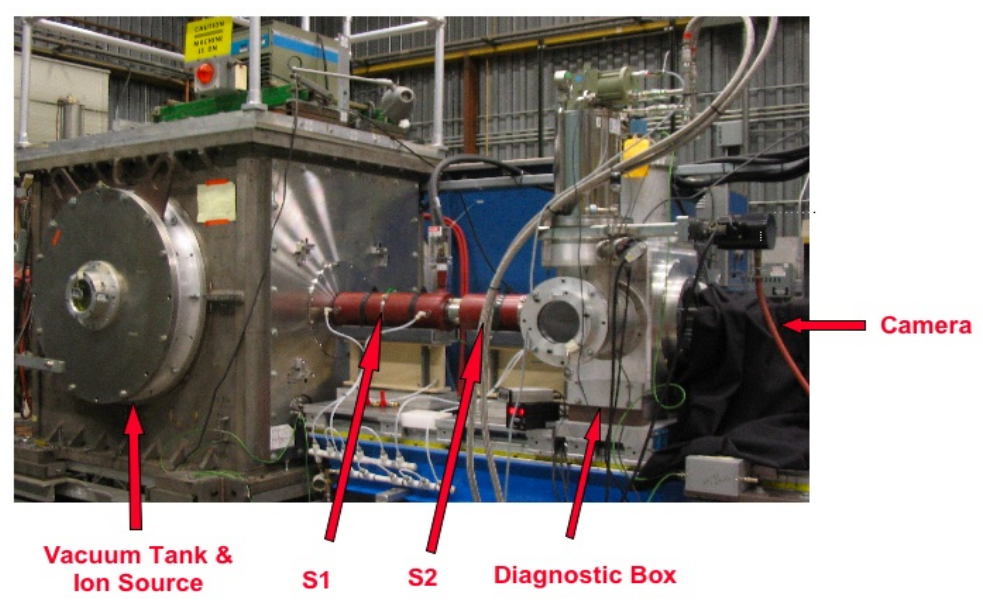

Figure 1.1: STX

The purpose of STX is to study the transport of the beam through solenoids, which act on the ion beam as converging lenses act on beams of light, preventing it from diverging due to 
space charge (which is the self repulsion of the positive ions). The principle is simple: when an ion with axial velocity enters the solenoidal field, it first "sees" a bump in the radial field, which gives it an azimuthal acceleration, and puts it on a quasi-corkscrew orbit. On such an orbit, the centrifugal force only depends on the speed, whereas the centripetal force is also proportional to the axial field. Thus, a strong field can overcome the divergence and has a focusing effect on the beam. When the beam exits the solenoid, the opposed radial field unspins the beam. Calculation of the solenoidal focal length can be found in [7]. Solenoids, together with quadrupoles, are key elements for the beam optics that are omnipresent in every particle accelerator.

The source is a ceramic placed at the anode, which heats up and evaporates $K^{+}$ions when a voltage is applied. The beam is a $300 \mathrm{keV}$, has an intensity of $45 \mathrm{~mA}$, and lasts $10 \mu \mathrm{s}$. It has been diagnosed as having a flat-top, and good emittance properties.

\subsection{Measurement of the eddy currents perturbation to the field}

In order to reach high enough magnetic fields for the solenoid transport, the magnets, nonsuperconducting, are pulsed with a strong sinusoidal discharge, of half-period $\frac{T}{2}=4 \mathrm{~ms}$.

The beam lasts only $10 \mu \mathrm{s}$ and passes through the solenoids when the field is at the top of the sine-wave, so that what it "sees" is a DC field of the peak value $(2.8 \mathrm{~T})$.

But the increase of the magnetic field, according to Faraday's law of induction, generates an electromotive force $e=-\frac{d \Phi}{d t}$, which, in the conductors submitted to the field, gives rise to eddy currents, that tend to create a field opposed to the increase in the magnetic flux.
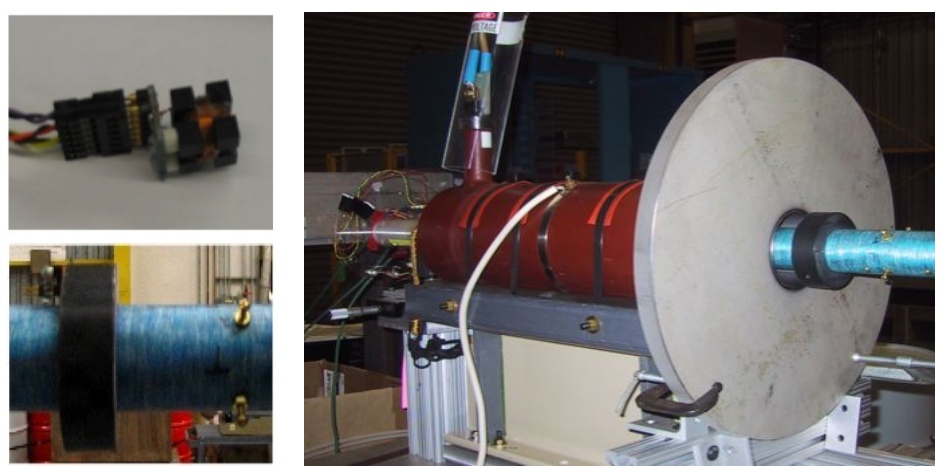

Figure 1.2: Experimental set-up for the magnets mapping

Top-left: the probes, composed of three coils each. Bottom-left: position of the on-axis and off-axis probe. Right: the bench with solenoid 2 and the stainless-steel plate

In STX, there are three regions where eddy currents can occur: the coil of the magnet itself, the beamtube, and the 2-cm thick stainless-steel plates near the source and diagostic chamber. Because they are much more massive, the end plates are expected to generate the dominant perturbation.

To measure the effect of the eddy currents the set-up shown in Fig. 2 was used. A probe containing on-axis and off-axis $(\mathrm{r}=3.53 \mathrm{~cm})$ copper coils is fixed while the experimentalist displaces the magnet in order to take data at different axial positions. The coils themselves measure the variations of the magnetic flux, and the same scans are done with and without 
the 2-cm thick stainless-steel disk which mocks up the real experiment. The plate is placed at the same distance from the magnet ends: $1.27 \mathrm{~cm}$.

Figure 3 shows $B_{z}$ as a function of time for several locations ( $\mathrm{z}=56$ to $60 \mathrm{~cm}$ ) slightly behind the plate $(\mathrm{z}=53$ to $55 \mathrm{~cm})$. In all these scans, the origin of the $\mathrm{z}$ axis is always taken at the lead end of the magnet.
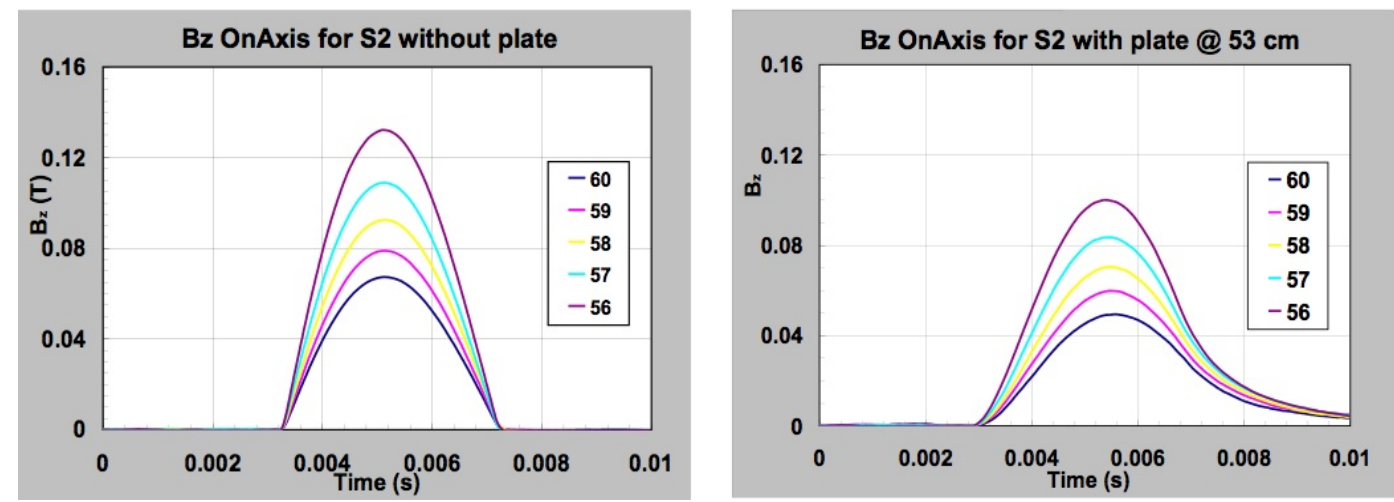

Figure 1.3: Effect of the eddy currents on the field wave-form near the plate

One can see that the waveform of the field without plate (left) is close to a half-sine wave, just proportional to the curent drive pulse, whereas with plate (right), the maximum value is lowered, and this maximum is delayed. What is important for the beam, is the value of the field at the time when it passes through the solenoid, which is considered to be the time of the maximum value of the drive current and of the field without plate ${ }^{1}$ and that we have been calling "beamtime".

Figure 4 shows the profile of $B_{z}$ on-axis at beamtime, with and without plate, and the ratio with/without. As expected, $B_{z}$ on-axis is reduced near the plate. The reduction is locally important (around 20\% near the plate and up to $40 \%$ passed the plate), but the global effect is not so important, because the effective length of the solenoid, $\int\left(\frac{B_{z}}{B_{\max }}\right)^{2} d z$, is only reduced by a factor of $1.4 \%$.

The same measurements have been done for the axial and for the radial field at $3.53 \mathrm{~cm}$ off-axis. Results are shown in section 1.4 comparing measurements with simulations.

\subsection{Modeling of the phenomenon}

All the physics of the eddy current effect can be derived from Maxwell's equations. In order to get good intuition of the phenomenon, it is useful to try to reduce the usual time-dependent system of PDEs to a small number of expressions describing the important phenomena for the experimentalist.

First model: the image recessing plate (Maxwell) A first attempt to grasp the physics of the eddy currents in the plates nearby a pulsed magnet, consists in approximating the

\footnotetext{
${ }^{1}$ In fact the maximum of the field, even without plate, is delayed by $\sim 60 \mu \mathrm{s}$ compared to the maximum of the current. This effect we have found to be attributable to the eddy currents in the beamtube.
} 


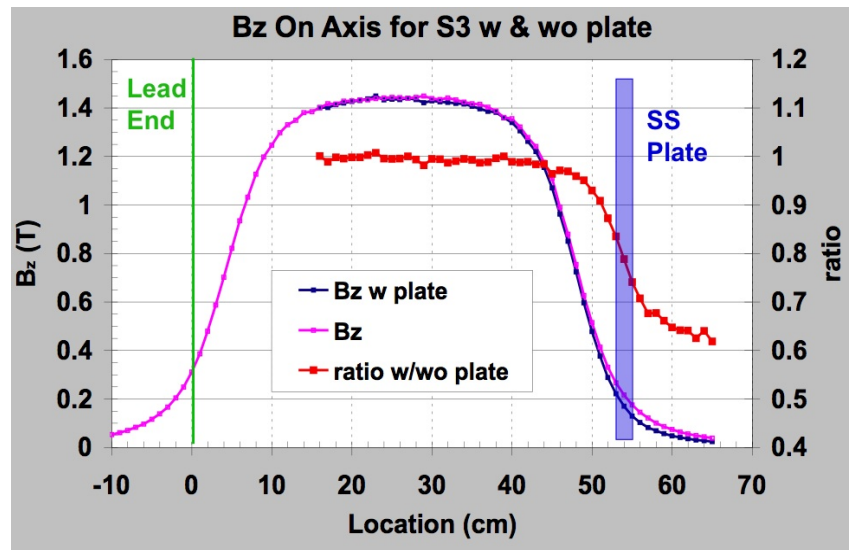

Figure 1.4: Reduction of $\mathrm{Bz}$ on-axis by the eddy currents The profiles with (blue) and without plate (purple) are taken at beamtime

metallic plate by an infinite thin plate without hole (three approximations at the same time). The solution for this problem has been calculated by J.C. Maxwell [8]. In this case, the exact solution for a sudden increase in the field (Green function) is given by adding to the solenoidal field the field of an image, symmetrical with respect to the metallic sheet plane, that recesses at a constant speed $v=\frac{2 \sigma}{\mu}$, where $\sigma$ is the surface resistivity of the plate and $\mu$ is its magnetic permeability.

I have made an implementation in Mathematica of that solution, that can be found in Appendix 1. Referring to the latter, one can see that Maxwell's solution describes well the reduction of $B_{z}$ far from the plate, but is not realistic close to the plate, because of the above mentioned approximations.

Exact analytical solution The exact solution for the the time-dependent electrodynamic problem with none of the approximations made above is not very simple, but the equations can be reduced to a simple set, making the intuition of the physics involved much easier than considering the four Maxwell's equations.

First, considering the cylindrical symmetry, we notice that the problem is completely described by the vector potential $\vec{A}=A_{\phi}(r, z, t) \vec{e}_{\phi}$ (only a $\theta$-component, with no $\theta$-dependence, see [9] for full explanation).

Second, see that Maxwell's equations reduce to a simple diffusion equation for the vector potential: $\frac{d \vec{A}}{d t}=\frac{\rho}{\mu} \nabla^{2} \vec{A}$, where $\rho$ and $\mu$ are the resistivity and the permeability of the conducting plate. ${ }^{2}$

Third, note that these equations, when applied to a thin sheet of metal, yield as a boundary condition that the impulsive response to a sudden change in the imposed field is: $A_{\text {eddy }}(t=$ $0)=-A_{\text {solenoid }}(t=0)$, which can be interpreted as an instantaneous, perfect shielding of the field by the eddy currents.

The global solution to our problem, then, is easy to picture (but not to calculate!): for every

\footnotetext{
${ }^{2}$ This equation is valid independently from the symmetry, and is the same that describes any skin effect in conductors submitted to varying fields.
} 
$\delta B_{\text {sol }}$ of increase in the solenoidal field, the plate reacts with eddy currents corresponding to a $\delta B_{\text {eddy }}$ exactly opposed to the former. Then, the vector potential diffuses through the plate, leading to a decay of the eddy currents.

The exact time-dependent solution could be computed with these equations, but, rather, we have implemented a finite element calculation that just solves all Maxwell's equations.

\subsection{Finite-element calculation with ANSYS and importation of the realistic fields in WARP}

After measuring and understanding the eddy-current perturbation to the solenoidal fields, the interesting step was to find a way to compute the effects and import them in the advanced particle-in-cell (PIC) code WARP, that is used by the HIF group to model most of the experiments.

Prior to this study, the magnetic fields that were used by WARP were calculated from a simple analytic, idealized, formula. All magnets were assumed to have identical field distribution, and no eddy currents were included. Given the level of accuracy that is needed globally on the NDCX experiment, it was decided that, even though the effect of the eddy currents had been shown to be globally small, it was useful to include more realistic fields in the code.

Therefore, the first step was to check the ability of a transient, finite-element calculation to reproduce the measured results. That is what I helped doing, together with Matthaeus Leitner who ran the code ANSYS, including one magnet and a metallic plate in its vicinity. Figure 5 and 6 show the very good agreement that was achieved. Several refinements in the mesh and adjustment of the geometrical parameters were required in order to match the ANSYS results with the measured field perturbations.
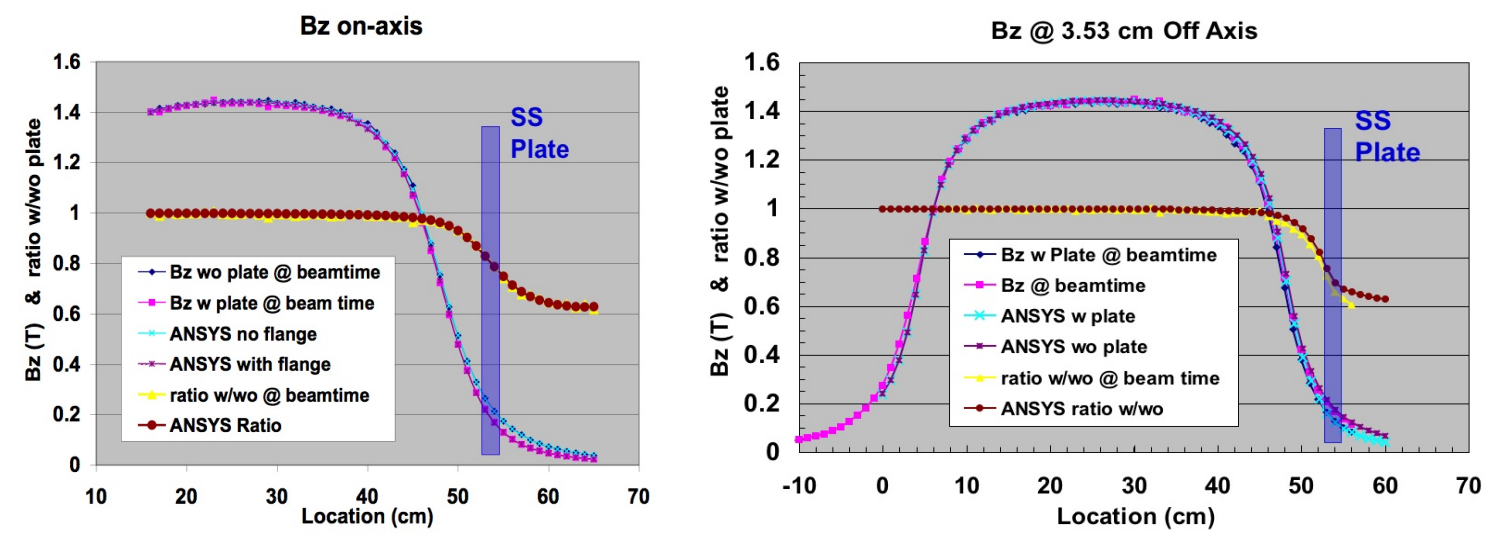

Figure 1.5: ANSYS simulation vs measurement for S3 (1)

An excellent agreement was obtained for $\mathrm{B}_{z}$ on-axis (Fig 5a), which is the most important function, as it is, on first order in $\mathrm{r}$, the field that actually focuses the beam. Both measurement (yellow) and ANSYS calculation (red) show a reduction of the field at beamtime of about $20 \%$ near the plate, and up to $40 \%$ behind the plate, and the error between ANSYS and the measured reduction is smaller than $1 \%$. 


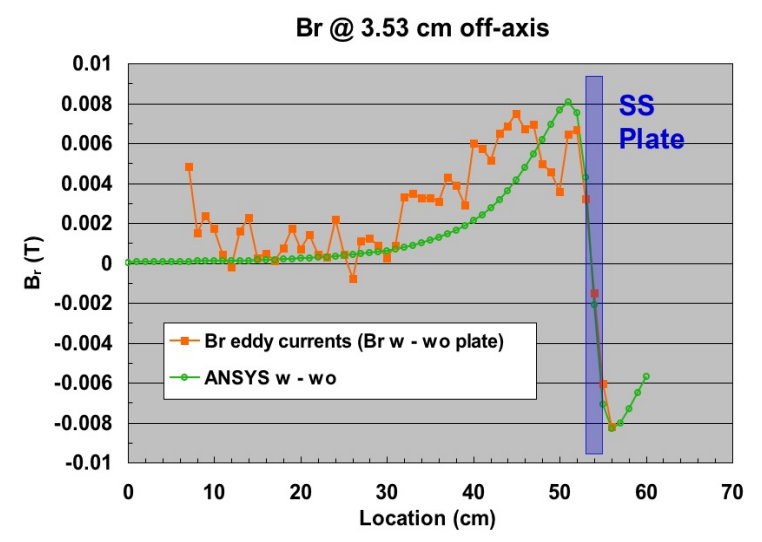

Figure 1.6: ANSYS simulation vs measurement for S3 (2)

For $B_{z}$ off-axis (Fig $5 \mathrm{~b}$ ), ANSYS has given good agreement with the measured reduction, but it is not as good as for $\mathrm{B}_{z}$ on-axis. The reason, though, has been considered lie in some discrepancies between the real magnet and the model magnet that is used in ANSYS. As can be seen in fig. 5.b, ANSYS without plate (purple) and $\mathrm{B}_{z}$ measured without plate (pink) do not coincide, which is very clear on the left part of the figure. The model magnet that is used in ANSYS had been adjusted so that its field on-axis fits the measured field on-axis. A full realistic model based on a global fit has not been done yet. It is then understandable that the agreement is not as good off-axis than on-axis.

Concerning $B_{r}$, which has much smaller values than $B_{z}$, what is shown in Fig. 6 , for the purpose of clarity, is the difference between the field with and without the plate, which thus is the field of the eddy currents at beamtime. The experimental noise is comparable to the values that are measured, but nonetheless one can see that ANSYS and the measurement are in good agreement, in particular in the vicinity of the plate, where a change of sign is observed.

Importation of the realistic fields in WARP Thanks to this study, and the effort to understand and reproduce the magnetic fields in detail, a much better knowledge of the magnets, their fields, and the perturbations by the eddy currents has been achieved. With the help of David Grote, a specialist of the WARP code, I have written a couple of small programs in Python that now allow the code to include realistic fields that we import directly from an ANSYS calculation.

In the last days of my stay, I did some runs with the new fields, but, unfortunately, some problems in the code, perhaps not even related to the importation of the fields, prevented me from being able to show a comparison between simulations with the old fields and the new realistic ones. In particular, we would have expected to see the reduction of the focusing power of a solenoid by 1 to $2 \%$ due to the eddy currents. Furthermore, non-ideal aspects of the fields may cause phase-space distorsion of the ion beam. 


\section{Chapter 2}

\section{WARP simulations of electron leakage due to diagnostics in STX}

One of the things I was very curious to learn about during my stay at LBNL was simulations. Since a campaign of simulations to study electron-cloud effects was planned to begin by the time I would be in the HIF group, it was decided that I would take part in it. The objective of this campaign (that is still going on) was to understand the mechanism of the failure of an electron trap observed in STX and, eventually, to find ideas about how to protect the beamline from this undesired leakage.

\subsection{Motivation: the experimental problem of the electrons leakage}

Electron clouds and how they are controlled in STX Electron clouds are a traditional enemy for every accelerator of positive ions. They can be generated by any interaction of the beam with elements of the beamline, and they often are attracted by the beam and can significantly perturb its properties. Their study is one of the strong focuses of the HIF group.

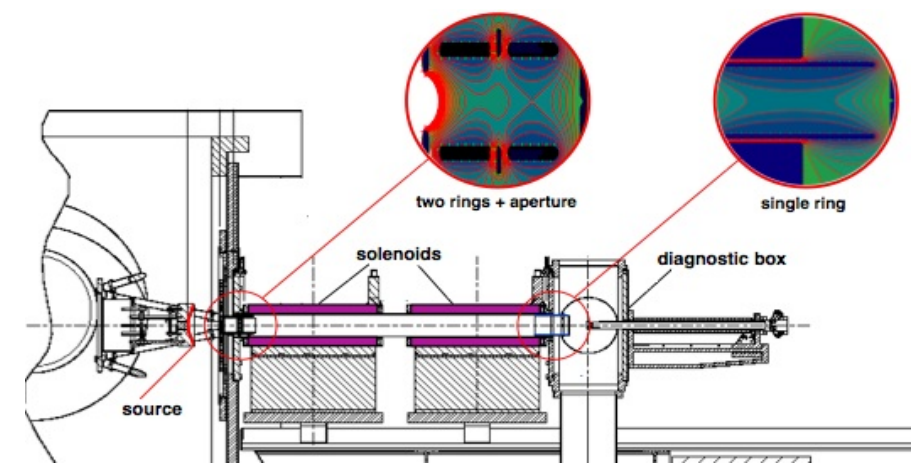

Figure 2.1: The electron traps in STX

Figure 1 shows the system to control electrons in STX. The electrons can come from the beam scraping the walls of the beamtube, from residual gas being ionized by the beam, or, 
most importantly, from the beam hitting metallic surfaces, like the aperture or the slit plate. The aperture is a metallic plate with a hole in its center that is placed little after the source to reduce the intensity of the beam (from $45 \mathrm{~mA}$ to $27 \mathrm{~mA}$ ). The slit plate is just a plate with a horizontal or vertical $0.1-\mathrm{mm}$ wide and 5 -cm long slit allowing ions to pass through, that is very frequently used for diagnostics, such as transverse profiles and phase-space measurements (done with with two consecutive slit plates with varying positions).

When the beam hits either the aperture or the slit plate, electrons, called "primaries" and neutral gas that is adsorbed on the metal are released. The so-called "neutrals" (gas atoms) can then be ionized if hit by the beam, which creates positive ions and more electrons, called "secondaries". The purpose of the electron traps is to prevent all electrons, which are much lighter and much more mobile than the ions, from invading the beamline.

Each electron-trap is a metallic ring biased negatively (typically $-3 \mathrm{kV}$ ) to create a potential barrier confining the electrons where they were produced. The two medallions in Fig. 1 show the disposition of the two electron traps: one, split into two halves, surrounds the aperture so that any electron remains close to it, going neither upstream in the source, nor downstream with the beam; the other, after the last solenoid, is supposed to prevent the electrons created at the slit-plate from leaking upstream.

The failure of the electron trap due to diagnostics in STX The system presented above has worked well most of the time, but recently, a failure of the final electron trap was observed in STX.
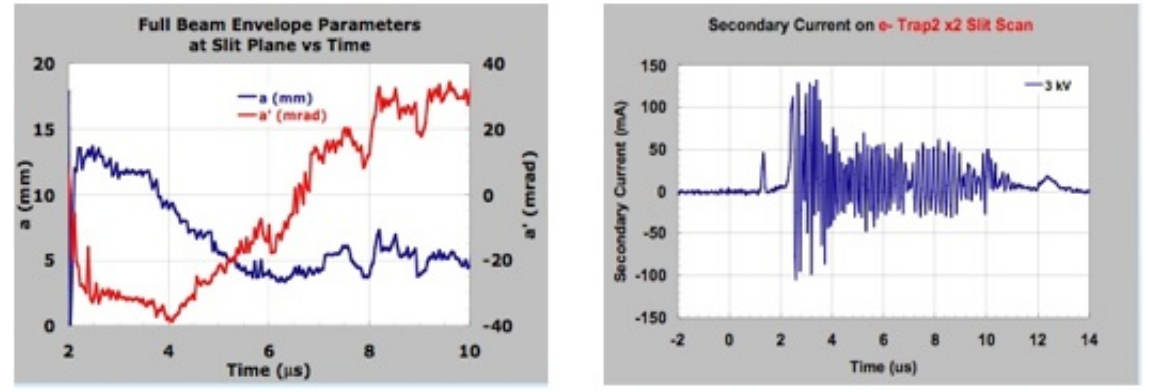

Figure 2.2: Experimental evidence of the electron leakage

Left: The radius (blue) and the rms envelope angle (red) are calculated from slit-plate diagnostics taken during the whole length of the beam. Right: Current signal

What the experimental team first observed was an undesired behavior of the beam envelope parameters, which are usually pretty constant all along the $10 \mu s$ duration of the beam. As can be seen on Fig. 2a, after less than $1 \mu \mathrm{s}$, the beam starts shrinking, its radius collapsing from $13 \mathrm{~mm}$ to $5 \mathrm{~mm}$. Also, the beam, initially converging, with a convergence angle $a^{\prime} \simeq-40 \mathrm{mrad}$, starts diverging while it contracts, reaching $a^{\prime} \simeq+35 \mathrm{mrad}$. Given the experience of the group in the subject, these results were interpreted as being caused by an electron leakage that partly neutralizes the space charge of the beam, causing its radius to shrink. Because the focal point of the beam is now before the diagnostics instead of being after, the beam is no longer convergent but divergent when it is diagnosed.

Fig. 2b shows an other characteristic diagnostic: the electron-trap current. At $1.4 \mu s$, when 
the beam passes in front of the final electron trap, a positive spike is observed, corresponding to the electrostatic response of the trap to the arrival of the beam ions. Then, one sees a positive build-up of positive current, until the time $2.2 \mu \mathrm{s}$, when wild oscillations begin suddenly, which are associated with electrons invading the beamline.

\subsection{Method for a study using WARP}

For the study of this problem, but also of general issues related to electron-clouds, the particlein-cell (PIC) code WARP has been updated, so that it can handle several species (beam ions, neutrals, primary electrons, ions, secondary electrons) [10]. The specialists of WARP have added modules to include the relevant physics of desorption, impact ionization of neutrals by the beam ions, and multi-species handling.

More precisely, these improvements had already been implemented and used for HCX (the other machine of the HIF group), but the cases that had been treated were quite limited, and the results had not really been compared to measurements. In that sense, the campaign of simulation that was triggered by the electron leakage, ended by being a very good opportunity for the team to test and improve the capability of simulating the physics of electron clouds.

Brief description of the code setting As usual in WARP, the particles that are pushed by the code are macroparticles, representing typically $10^{6}$ to $10^{9}$ real particles each. This method is very well suited for the simulation of the high current beams that are studied by the HIF group, because such a scheme naturally leaves apart most of the particle-particle coulombian interactions, and emphasizes more the collective effects. As they move faster, the electrons are pushed with a time step 5 times shorter than the one for heavier particles.

Some parameters had to be chosen, sometimes with little certainty or justification (see section 2.4). For the desorption of neutrals, when a beam ion hits a metallic plate at normal incidence, it was chosen that $10^{4}$ neutrals are released. The species of gas desorbed was finally chosen to be $\mathrm{H}_{2}$, after some tests showing that heavier species $\left(\mathrm{H}_{2} \mathrm{O}\right.$ or $\left.\mathrm{CO}_{2}\right)$ were not giving the oserved onset time for the beginning of the electron leakage, and also because stainless-steel is known to preferentially absorb $\mathrm{H}_{2}$. The cross section for impact ionization of the neutrals by the $300 \mathrm{keV} K^{+}$beam ions was taken to be $5 \cdot 10^{-20} \mathrm{~m}^{2}$.

\section{$2.3 \quad$ Results of the simulations}

It took some time to prepare the code, and several times during the campaign something in WARP needed to be fixed. Despite these problems, a series of runs was completed, mostly by Bill Sharp, and also by me, which allowed to get good qualitative understanding of the problem under study.

Observation of the electron leakage We simulated two different situations: the slit plate for diagnostics placed either directly after the source and aperture with electron trap, or after two solenoids and electron trap.

As in the experiment, the case with no solenoids (Fig. 3a) showed no electron leakage. After the beam hits the slit plate, many neutrals are desorbed (left), $\mathrm{H}_{2}^{+}$ions and electrons are produced (center and right) and the ions are attracted to the negatively-biased electron 
trap (center), but the electron-trap works, and the primary (blue) or secondary electrons (red) produced near the aperture or slit plate stay confined in their vicinity (right), even after $4 \mu s$ of simulation.
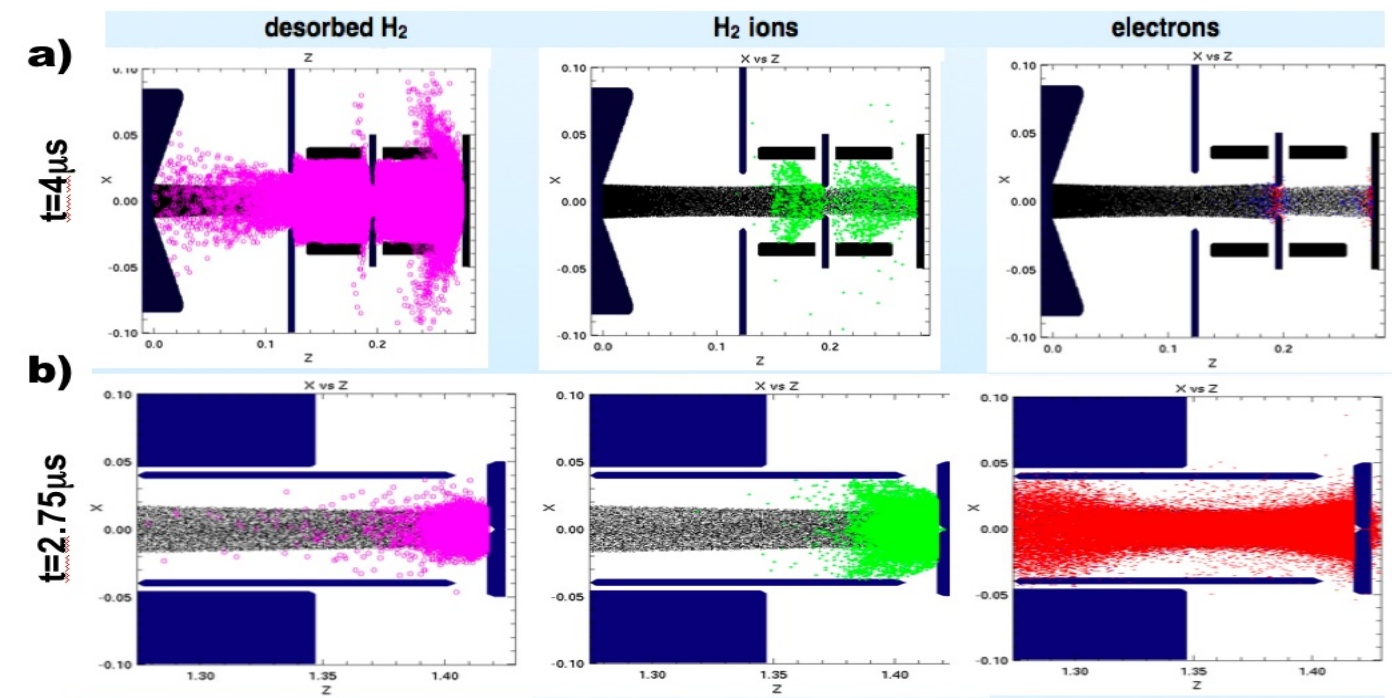

Figure 2.3: Behavior of the different species

a) After source and aperture limiting the current (beam ions in black) b) After two solenoids

On the contrary, in the case after solenoids (Fig. 3b), one sees the leakage of electrons starting at $2.75 \mu \mathrm{s}$ already, which is $1.3 \mu \mathrm{s}$ after the beam has hit the plate. The $\mathrm{H}_{2}^{+}$ions are attracted to the electron trap (center), whereas the corresponding electrons sit in front of the slit plate and start building-up a negative potential. When this potential is big enough to overcome the $-3 \mathrm{kV}$ bias of the electron-trap, they are pushed beyond the failing barrier, and invade the beamline (right).

The oscillations of relaxation Thanks to diagnostics performed at different times (every $25 \mathrm{~ns}$ only for the moment), we have been able to reconstruct more precisely the sequence of the phenomenon, i.e., to observe the periodical detachment of "bunches" of electrons.
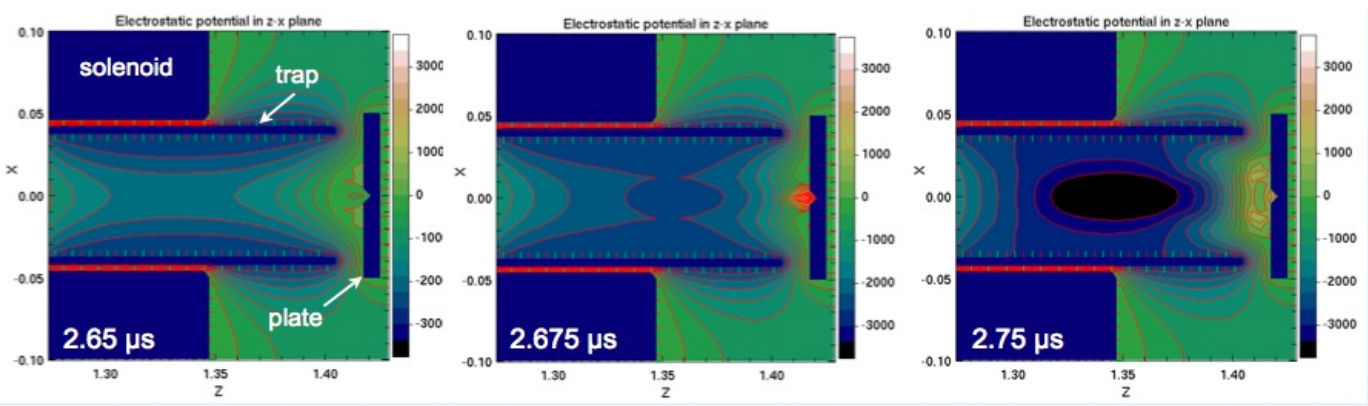

Figure 2.4: Oscillating leakage 
Figure 4 shows, with not a very good time-resolution, the different moments in the detachment of an electron bunch, which is the first one that our sampling allowed to observe. The plot shows a map of the electrostatic potential before (a), after (b) and during the detachment of a bunch of electrons (c). The reversed chronological order leads us to suspect that the frequency of our sampling is not high enough, otherwise we should see a leakage like (c) between (a) and (b), the reason being that the build-up of a positive potential near the plate, as seen in (b), can only occur after the leakage of some electrons.

Figure 5 shows the plot that contains all the information together: the electron-trap current signal. The agreement is qualitatively good between the experiment (a) and WARP (b). In both pictures, a spike is observed when the beam enters the trap. Then, a positive current builds-up gradually, due to positive ions reaching the trap, and, after a time that is not the same in both cases, oscillations begin. Concerning the period of the oscillations, a detailed analysis has not been done yet, but it is clear that WARP and the experiment coincide on something in the range of 10 to $100 \mathrm{~ns}$.
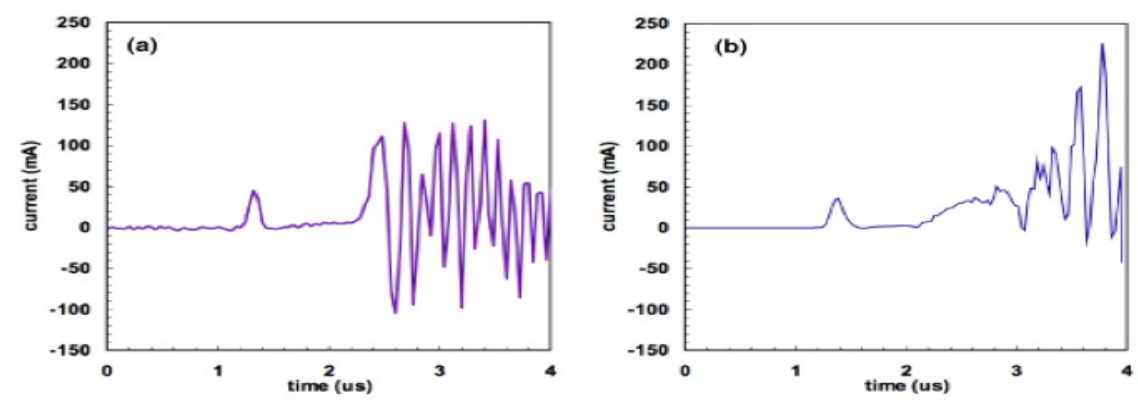

Figure 2.5: Electron-trap current measured (a) and calculated by WARP (b)

\subsection{Summary and perspectives}

This simulation campaign has given good qualitative results, but big uncertainties remain, in particular concerning the values of the parameters for the desorption of neutrals and the impact-ionization cross section, as well as the nature of the desorbed gas. A more detailed critical review of these problems can be found in [11]

It is important to say here that some other puzzles, related to discrepancies between WARP and the experiment, are still remaining. For example concerning the dependence of the leakage on the electron-trap voltage, the onset time for the lekage, or even the simple fact that, experimentally, when the diagnostics are moved $20 \mathrm{~cm}$ downstream (in the case after solenoids), the leakage is not observed! WARP was not able yet to reproduce this result. All these issues currently the modeling team from really trusting any of the WARP predictions.

Much has been learnt already, especially by the WARP-development team, but it is still work in progress. 


\section{Chapter 3}

\section{Hydrodynamics of the metallic 2-phase flow in upcoming target experiment}

The last project in which I was involved is the one in which I had most the exciting impression of doing innovative research. First, the plan was that, together with John Barnard and Dick More, I would analyze some hydrodynamic simulations of upcoming target experiments. But, progressively, I focused on new questions about surface effects, and we took on new paths in order to build a qualitative description of the 2-phase flow and do some predictions for the droplet size and evolution.

\subsection{The context: target experiments and hydrodynamic simu- lations without surface effects}

WDM research In the coming year, the NDCX beam, that should be compressed both longitudinally and transversely, will be used for target experiments, with the purpose of studying the Warm Dense Matter regime (densities from 0.1 to $10 \mathrm{~g} / \mathrm{cm}^{3}$ and temperatures from 0.1 to $1 \mathrm{eV}$ ). In these regimes, the equation of state (EOS), i.e. the functional relation between $\mathrm{P}, \rho$ and $\mathrm{T}$ (a surface in the 3 -D space of those parameters) is not very well known, principally because these regimes lie just in between the solid $(\mathrm{T}=0)$ and the plasma $(T>>1 \mathrm{eV})$ domains, for which good and relatively simple theoretical models are available. On the contrary, in the WDM range, phenomena such as the strong coupling ${ }^{1}$ are not well understood yet [12]. The simulations use several different EOS tables, each one based on some combination of data and formulas. But experiments are still necessary to discriminate which EOS are good.

Among the planned experiments are the foil heating experiments. These consist in focusing a mm-sized intense ion beam onto an order of micron-thin metallic foil (or foam), heating it to the eV-range $(1 \mathrm{eV}=11,605 \mathrm{~K})$ in a time that should be short enough $(\sim 1 \mathrm{~ns})$ so that the hydrodynamic expansion of the liquid (or vaporized) metal does not prevent it from reaching the desired temperatures.

\footnotetext{
${ }^{1}$ This name is given to a regime where the potential energy between ions is ion the same order or greater than their kinetic energy.
} 
Prior to the experiments, a large number of simulations have been carried out in order to predict the phenomena that should be observed, and to optimize the design of the experiments for the purpose of gaining knowledge about the EOS in the WDM regime. All the work I did in that project is also part of this theoretical effort, which is comprehensively reviewed in [13].

Simulations without surface effects When I arrived, a nominal (i.e. reference) case had already been chosen and simulated. The nominal case uses a $\mathrm{Li}^{+}$beam of energy $2.8 \mathrm{MeV}$ per ion, with a pulse length of $1 \mathrm{~ns}$, hitting a $3.5 \mu \mathrm{m}$-thick foil of aluminum. The beam enters the foil somewhat above the Bragg peak (maximum of the energy deposition $\mathrm{dE} / \mathrm{dx}$ ), and leaves it somewhat below it, so that the energy deposition in the foil is uniform within $10 \%$ variation $^{2}$. As the heated volume is much thinner than it is wide (the focal spot has a radius of $1 \mathrm{~mm}$ typically), the geometry is one-dimensional, at least for the first part of the expansion.
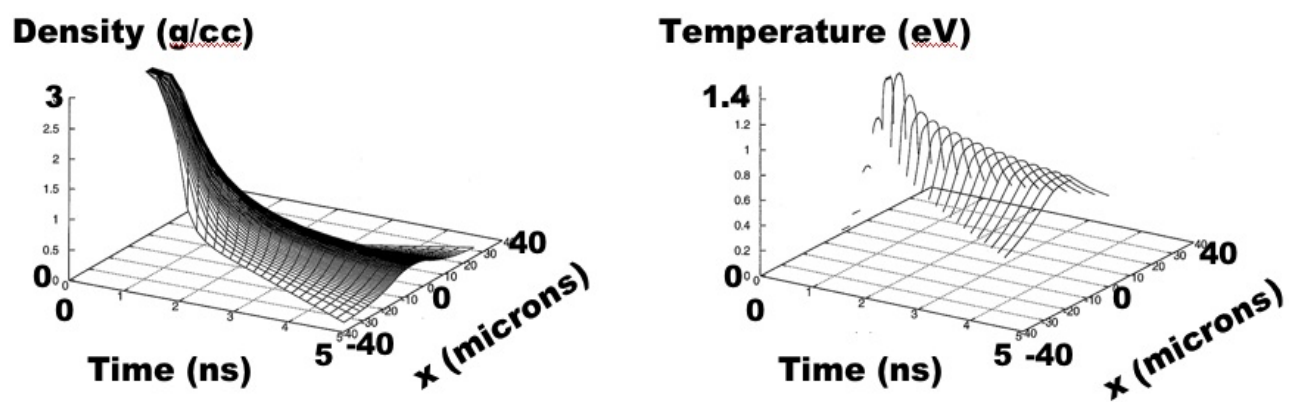

Figure 3.1: Expansion and cooling of the foil (nominal case)

Figure 1 shows a result of a DPC simulation that was carried out by Dick More. The code is a simple 1-D hydrodynamic lagrangian code, that uses for the equation of state a table that is constructed from known values and interpolations, and based on the Saha equation [15]. On these two pictures, one sees the decay of the density (a) and the rise and decay of the temperature (b) in space and time. During the first ns, the foil expands from its initial $3.5 \mu \mathrm{m}$ thickness, and gets heated. After $1 \mathrm{~ns}$, the heating pulse has ended so there is only adiabatic expansion and cooling of the foil.

The first task that was assigned to me was to analyze in detail the evolution of the center temperature and density, in the different cases that were run (with variation of the thickness of the foil, the pulse length, and the energy deposition). By plotting these functions in log-log plots, I found that, after the end of the pulse, T decays following two power laws (approximately $-2 / 3$ and then approximately $-1 / 3)$ while $\rho$ seemed to decay with only one power law $(-1)$.

There was no real interpretation for these power laws, but the discontinuity in the decay of $\mathrm{T}$ was found to always correspond to the time when the center of the foil, after having melt because of the heating, enters the 2-phase region (see the phase diagram in Fig. 2 below). This, added to a certain uneasiness in applying hydrodynamic equations to a flow that becomes inhomogeneous, led us to a questioning about the structure of the gas and liquid phases.

\footnotetext{
${ }^{2}$ This ability to heat volumetrically and nearly uniformly is one of the important advantages of heavy ion beams as drivers for warm dense matter studies (see [14] for example).
} 


\subsection{How can we describe a 2-phase flow?}

In the DPC code, the EOS uses the maxwellian construction, which means that, if one computing cell is in $\rho-T$ conditions corresponding to a mixture of liquid and gas, the associated pressure corresponds to the equilibrium configuration with just one flat interface between some gas (usually on top because of gravity) and some liquid. This may not be bad for the calculation of the macroscopic hydrodynamics, but it does not say anything about the microphysics of the bubbles and/or droplets.
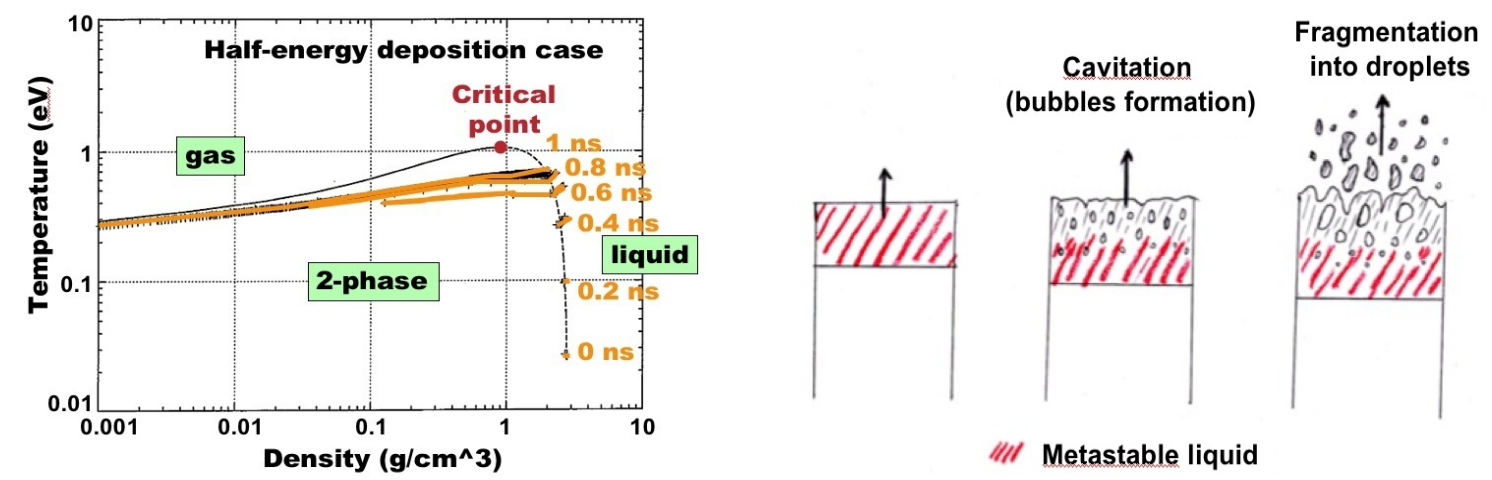

Figure 3.2: Evolution of the foil in the $\rho-T$ plane

Figure 2a shows the phase diagram taken from the EOS used in the DPC code, together with the trajectory of the foil in the $\rho-T$ space, for the half nominal-energy deposition case. The black line corresponds to the boundary between the liquid, gas, and 2-phase zones. Each orange line describes the state of the whole foil at a certain time: at each time, the right-hand edge (high $\mathrm{T}$ and $\rho$ ) corresponds to the center of the foil, and the left-hand edge to any of its borders (the simulation is symmetrical by change of sign of $\mathrm{z}$ ). At $0 \mathrm{~ns}$, the foil is at room temperature $(0.025 \mathrm{eV})$ and solid density $\left(27 \mathrm{~g} / \mathrm{cm}^{-3}\right)$. Then, it is heated, reaching $0.7 \mathrm{eV}$ in the center, and then $\mathrm{T}$ and $\rho$ decay because of the expansion, called rarefaction wave.

According to this diagram, the foil first melts nearly completely, and then enters the 2phase region. The liquid thus becomes thermodynamically unstable, and tends to evaporate, which means that what is likely to happen is that bubbles of gas nucleate in the over-expanded liquid and, at some point, the liquid fragments into droplets, as pictured in Fig. 2b.

Bubbles or droplets? We have proposed an answer for this question about the nature of the flow. If more than half of the volume of the fluid is liquid, we postulate that the flow is composed of small bubbles in a continuous liquid phase. If less than half of the volume is occupied by liquid, then we consider it to be a a flow of droplets in a continuous gas phase. The criterion to distinguish the bubbles or droplets regime is simple: the border can be represented in the phase diagram by the line of average density between liquid and gas phases (see red line on Fig. 3a).

In the nominal case, the simulations have shown that after $\sim 1.6 \mathrm{~ns}$ the whole foil has a density lower than $1 \mathrm{~g} / \mathrm{cm}^{3}$, which, using our criterion, means that all the material is in the droplets regime. The implication is that most of the flow can be described as a cloud of 


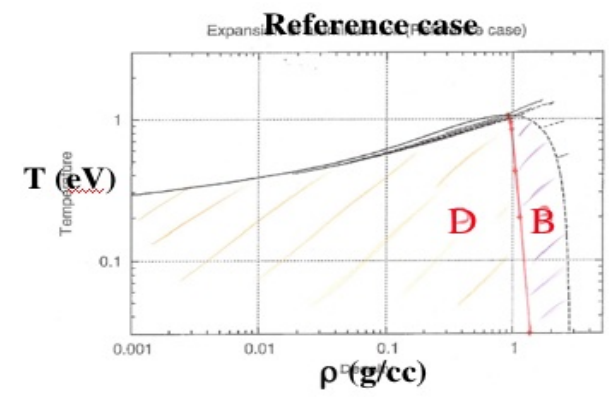

Figure 3.3: The bubbles and droplets sub-zones inside the 2-phase region

droplets. Now, the questions that come to mind are: what are their sizes, and how do they evolve?

\subsection{Maximum size of droplets in an expanding flow}

Considering the sketch of the fragmentation in droplets (Fig. 2b), one could think that what should determine the size of the droplets is the distribution of the bubbles when the fragmentation starts happening (i.e. when the condition of half volume in gas phase is reached). However, our approach has been to consider that, in an expanding flow such as predicted by the simulations, there may be a maximal size for stable droplets, determined only by the hydrodynamic disruptive and restoring forces acting on each droplet.

a)

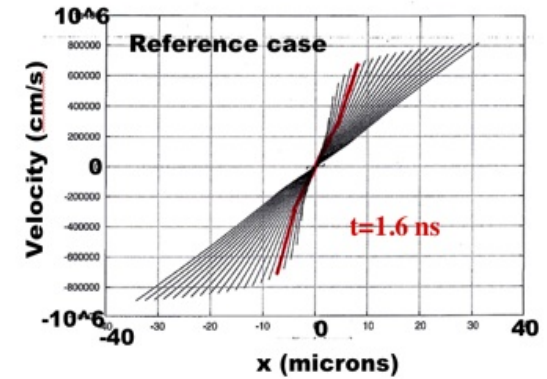

b)

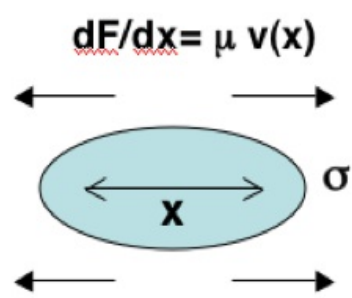

Figure 3.4: Expanding flow and stretched droplet

Figure $4 \mathrm{a}$ shows the velocity profiles at times from 0 to $5 \mathrm{~ns}$ in the reference case. The velocity of the material is nearly linear as a function of position (Hubble flow), especially for the longer times. ${ }^{3}$

Capillary number approach Our first idea was to write the conditions of stability for a steady droplet that is submitted to a stretching force from the surrounding, expanding gas, and to the surface tension acting as a restoring force (see sketch on Fig. 4b). If $\mathrm{x}$ is the dimension of the droplet, the stretching force is $\int F(x) d x=\int \mu v(x) d x=\int \mu \frac{d v}{d x} x d x \sim \mu \frac{d v}{d x} x^{2}$, whereas the surface tension force has to scale like $\sim \sigma x$. If one equates the two forces, the condition 
obtained for the equilibrium is that the capillary number $C a=\frac{\text { viscosity }}{\text { surface tension }}=\frac{\mu \frac{d v}{d x} x}{\sigma} \sim 1$, which yields a maximal size $\mathbf{x}_{\max }=\frac{\boldsymbol{\sigma}}{\boldsymbol{\mu} \frac{\mathrm{dv}}{\mathbf{d x}}} \cdot{ }^{4}$

After estimating the surface tension and the viscosity of aluminum in the appropriated ranges (see Appendix 2), we were able to estimate the maximal size of the droplets that appear in the middle of the foil at the time when it enters the droplet regime (1.6 ns). Considering typical droplet formation conditions as being around $\mathrm{T}=0.9-1 \mathrm{eV}$ and $\rho=1 \mathrm{~g} / \mathrm{cm}^{-3}$, we take $\sigma=100 \mathrm{dyn} / \mathrm{cm}, \mu=5 \cdot 10^{-3} \mathrm{~g} \cdot \mathrm{cm}^{-1} \mathrm{~s}^{-1}$ (viscosity of the gas calculated from kinetic theory), and a stretching ratio $\frac{d v}{d x}=10^{-9} s^{-1}$ (taken from the velocity profile in the DPC simulation), which finally yields $x_{\max }=200 \mathrm{~nm}$.

Weber number approach Another approach we developed consists in considering the balance between a disruptive dynamic pressure due to the velocity gradient inside the droplet, and the restoring surface tension. In this approach the droplet is not supposed at rest, and the disruptive term comes from inside it, from its inertia, not from the surrounding gas. A maximal size is found by equating the total dynamic pressure $\rho v^{2} A=\rho\left(\left(\frac{d v}{d x}\right) x\right)^{2} x^{2}=\rho\left(\frac{d v}{d x}\right)^{2} x^{4}$ with the surface tension $\sim \sigma x$, which corresponds to a Weber number $W e=\frac{\text { inertia }}{\text { surface tension }} \sim 1$. We obtain $\mathbf{x}_{\max }=\left(\frac{\sigma}{\rho\left(\frac{d \mathbf{v}}{\mathrm{dx}}\right)^{\mathbf{2}}}\right)^{\frac{1}{3}} \cdot{ }^{5}$

Applying this formula to the same situation as with the capillary number, and taking $\rho=1 \mathrm{~g} / \mathrm{cm}^{-3}$ for the liquid, we obtain $x_{\max }=50 \mathrm{~nm}$.

The capillary and the Weber number approaches yield a similar value for the maximal size of a droplet formed in the middle of the foil in the reference case. Their difference by a factor of four does not imply that a choice should be made; we do believe that both calculations have some relevancy (even though the capillary number calculation seems more directly relevant). If both calculations were "true" though, the minimal of the two maximal sizes would be the important one. But, as these two calculations are based on order of magnitude analysis, with no geometrical factors taken into account, it should be considered that, in this case, they coincide in saying that the maximal size of the droplets is of order $100 \mathrm{~nm}$.

\subsection{Kinetic model for the evaporation of a droplet}

After stating that the expanded liquid must fragment into 100-nm droplets, it is natural to investigate what happens to them. During the expansion, $\mathrm{T}$ and $\rho$ decrease, so $\sigma$, which is a decreasing function of $\mathrm{T}$, increases. The kinetic viscosity, proportional to $\sqrt{T}$ only (see Appendix 2), decreases. And the stretching factor $\frac{d v}{d x}$ decreases too (see profiles on Fig. 4a). Consequently, the maximal size due to hydrodynamic disruptive forces, calculated either as $x_{\max }=\frac{\sigma}{\mu \frac{d v}{d x}}$ or as $x_{\max }=\left(\frac{\sigma}{\rho\left(\frac{d v}{d x}\right)^{2}}\right)^{\frac{1}{3}}$, does increase during the expansion, which means that the

\footnotetext{
${ }^{4}$ In some bibliographical research I found that the exact same formula (but in cases of isothermal extensional flow) has been used by several authors (see [17], [18]). They also have the same sketch for the drop!

${ }^{5}$ Other authors have shown that the Weber number controls the maximal size of droplets that are in uniform motion relative to a surrounding fluid (see [19]), but this is not directly analogous to our situation.
} 
first generation of droplets is not bounded towards growth. However, we would rather expect each droplet to evaporate, as the density of the gas falls down dramatically in the expansion.

Description of the model In order to give more detailed answer to this puzzle, we have developed a model, that I have written in Mathematica, for the evolution of a droplet in an expanding flow. The model takes four variables: $N_{l}, T_{l}, N_{g}, T_{g}$ (number of particles and temperature of the liquid and the gas), and it solves four differential equations:

1) $\frac{d\left(N_{l}+N_{g}\right)}{d t}=0 \quad($ mass conservation $)$

$2) \frac{d N_{l}}{d t}=\beta\left(-\Phi_{v a p}+\Phi_{c o n d}\right) \quad$ (evaporation and condensation on the droplet)

3) $\left.\frac{d N_{l} c_{v g} T l}{d t}=\beta\left[-\left(L_{0}+c_{v g} T_{l}\right) \Phi_{v a p}+\left(L_{0}+c_{v g} T g\right) \Phi_{c o n d}\right)\right]+(1-\beta) \alpha \Phi_{c o n d} c_{v g}\left(T_{g}-T_{l}\right)$

(energy equation for the liquid)

4) $\frac{d N_{g} c_{v g} T g}{d t}=\beta\left[c_{v g} T_{l} \Phi_{v a p}-c_{v g} T g \Phi_{\text {cond }}\right]-(1-\beta) \alpha \Phi_{\text {cond }} c_{v g}\left(T_{g}-T_{l}\right)+\frac{\partial E_{g}}{\partial V_{g}}\left(\frac{\partial V_{g}}{\partial t}+\frac{\partial V_{g}}{\partial N_{g}} \frac{\partial N_{g}}{\partial t}\right)$ (energy equation for the gas)

These equations are a non-equilibrium model based in the first place on the expression of the evaporation and condensation fluxes from the kinetic gas theory: $\Phi_{\text {cond }}\left(T_{g}, N_{g}\right)=\frac{N_{g}}{V_{g}}\left(\frac{k_{B} T_{g}}{2 \pi m}\right)^{\frac{1}{2}} S_{l}$, where $S_{l}$ is the surface area of the droplet. The evaporation flux of some liquid at $T_{l}$ is calculated by saying that it is exactly opposed to the condensation flux from a virtual gas that would be in equilibrium with this liquid (i.e., the kinetic evaporation flux for a gas at the temperature $T_{l}$ and at the equilibrium density $\left.n_{\text {sat }}\left(T_{l}\right)\right) .{ }^{6} \beta$ is a sticking coefficient $(0<\beta<1)$ that represents the proportion of colliding atoms that effectively stick and condense onto the liquid.

At any temperature, the densities of the liquid and the gas are taken from the phase diagram of the EOS used in the DPC code (cf. Fig. 2a), at the boundaries with the 2-phase region.

In (3) and (4), the energy equations, the first three terms are nearly the same (but opposed), corresponding respectively to the evaporating, condensating and non-sticking collisions. The only difference is that for the liquid energy equation (3), each evaporating or condensating atom carries $L_{0}$, the latent heat at $\mathrm{T}=0 \mathrm{~K}$ (see Appendix 2), and not in the gas energy equation. That is due to the way we count the energies, with ideal specific heats taken as $c_{v g}=\frac{3}{2} k_{B}$ and $c_{c v l}=3 k_{B}$.

In (3) and (4), the third term, describing thermalization, is proportional to $(1-\beta) \Phi_{\text {cond }}$, the flux of atoms that collide but do not stick, and also to the difference $T_{g}-T_{l}$ (we chose this form because it is the simplest). $\alpha$ is a thermalization coefficient $(0<\alpha<1)$ controlling the magnitude of the effect.

\footnotetext{
${ }^{6}$ In fact, their is a subtlety: the equation for the evaporation flux includes Kelvin's equation, accounting for all surface effects: $\frac{n_{\text {sat }}(\text { curved })}{n_{\text {sat }}(\text { flat })}=\exp \left[\frac{2 \sigma}{n_{l} k_{B} T_{l} R_{l}}\right]$ (where $R_{l}$ is the radius of curvature of the liquid). This equation expresses enhancement of the evaporation for small sizes, but its effects have not shown to be significant for the evolution of the droplets: the maximal enhancement of the evaporation flux is by $\sim 50 \%$, when the droplet is 10 atoms big...
} 
Another assumption of the model is that an atom evaporating from the liquid has the same temperature as the liquid. Without going into the details of cutting a Maxwell-Boltzmann distribution at $L_{0}$, and renormalizing the new distribution, I wlll just say that it is only an approximation, but it is very good most of the time.

In addition to the energy carried by the particles, the gas energy equation (4) includes an adiabatic compression term, caused by two phenomena: the overall expansion of the cell, and the compression due to the apparition of some new gas from the liquid (last term). We have not written any corresponding compression/expansion term in the liquid energy equation (3), because it has been considered much less important, as the changes in the liquid density are very limited.

Results of the code Figure 5 shows the evolution in time, during 50 ns, that was obtained for a droplet of volume $(100 \mathrm{~nm})^{3}$ surrounded by the same volume of gas. We initialize the code in conditions of thermodynamical equilibrium $\left(T_{l}=T_{g}\right)$, and then we let the system evolve, with a volume expansion $\mathrm{V}(\mathrm{t})$ imposed from outside, linear with a coefficient $\frac{d v}{d x}=10^{-9} \mathrm{~s}^{-1}$ (taken from the DPC simulation). We choose (arbitrarily) $\alpha=\beta=0.5$.
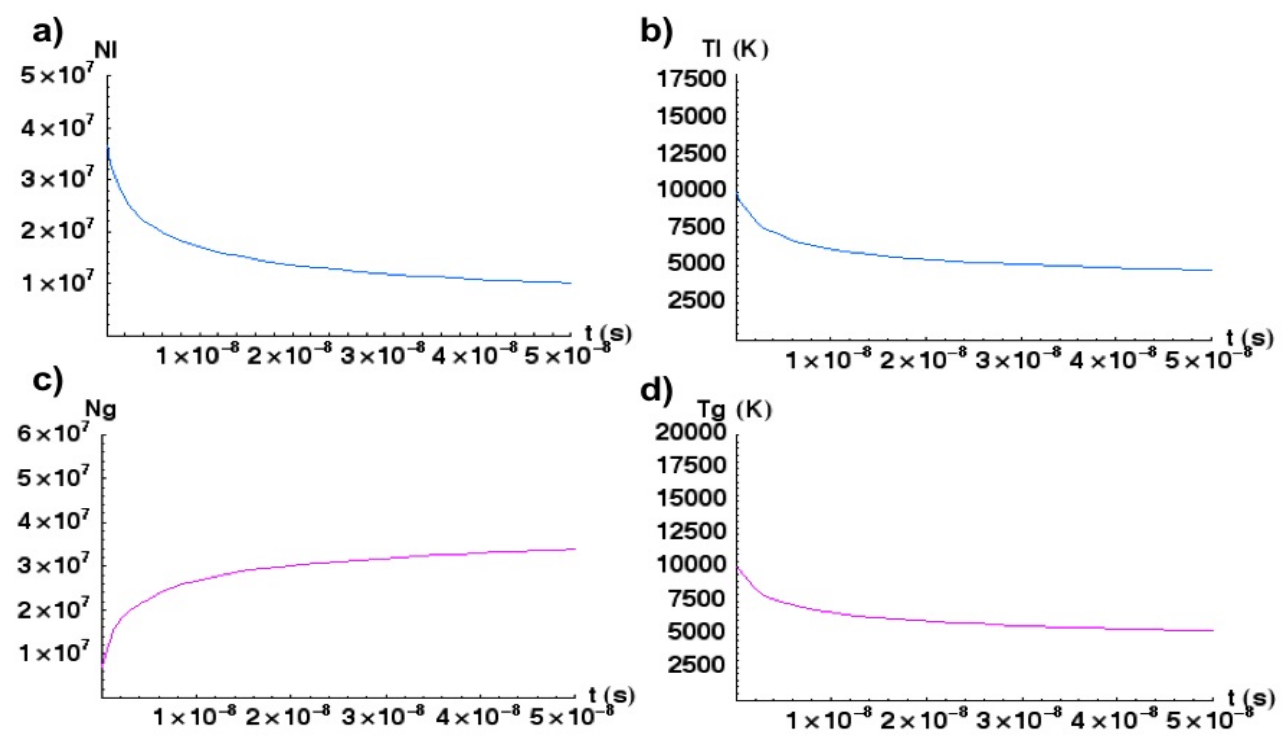

Figure 3.5: Evolution of a cell of liquid and gas in an expanding flow

The result shows that, in the first $20 \mathrm{~ns}$, about two-thirds of the liquid evaporates $\left(N_{l}\right.$ goes from $3.510^{-7}$ to $1.210^{-7}$ ) but, after that time, the evaporation becomes very slow. Further tests have shown that this is due do the cooling. Once the temperature of the liquid has fallen down enough, the evaporation becomes very difficult, because the latent heat $\mathrm{L}(\mathrm{T})$, which measures the deepness of the potential well from which an evaporating molecule has to escape, has become much bigger than the average energy $k_{B} T_{l}$ (cf. Appendix 2).

The result of this code has shown to be dependent on the values of $\alpha$ and $\beta$. For example, if there is no thermalization $(\alpha=0)$, and no heat exchange from liquid to gas via particles exchanges (but with no net flux $(\beta=0)$ the droplet will simply "freeze" at a certain size, 
because the evaporation can strictly not go any further than the point when all the energy of the initial liquid has been "consumed" in latent heat for evaporation. This case, though, is not very representative because it implies to assume $\beta=\alpha=0$, which means considering the case of an isolated droplet evaporating in vacuum, which the model has shown to be a bad approximation.

The run presented here shows droplets still existing after $50 \mathrm{~ns}$ (during which the material has travelled $0.25 \mathrm{~mm}$ ), and other runs we did let us think that the droplets might survive even after several $\mathrm{cm}$ of expansion, and perhaps, hit the walls of the target chamber. So, the conclusion is that, unless our choices were very unrealistic, in probably large ranges of parameters, one should expect to observe nano-droplets of liquid metal in the upcoming foil heating experiments. 


\section{Conclusion}

On the whole, I consider that my experience at LBNL was very interesting and fruitful. I have the impression of having learnt a lot, in fundamental domains such as physics of beams and plasmas and statistical thermodynamics, but also in applied fields such as experimental methods for accelerator physics and diagnostics. I also learnt about the computational and simulation tools that are used omnipresently in plasmas physics to simulate the systems in parallel with the experiment. In particular, I had the opportunity of learning a little bit the object-oriented language Python (used to operate WARP), and a good deal of Mathematica.

The three projects that I got embarked in gave me a lot of things to do, and generated a lot of interactions, very stimulating, with the other members of the group. It may have been ambitious to take on the three of them, but this combination was also very interesting because it allowed me to participate in most stages of the physics done by the HIF group, from the acceleration and the beam optics to the simulations and the plans for target experiments.

Concerning the eddy currents project, I have the impression that we achieved quite a complete solution to the problem: the eddy currents were measured, characterized, understood theoretically and reproduced by simulations. And now these results are being imported in WARP, for more realistic simulations.

Concerning the electrons project, the conclusion can certainly not be as round and smooth. The code is still being improved, and is not yet really reliable. The results of the simulations were a good achievement though, because they gave good qualitative insight in the physics of the electron leakage. But some improvements are really needed, like the time resolution that should be made shorter in order to really resolve the oscillations. Also, several input parameters need to be inquired and justified.

Concerning the two-phase hydrodynamics project, which consisted mostly in predictions, the results need to be compared to the experiments. Still, I think that the description of the two-phase flow that we elaborated has some self-consistency already. The qualitative description and the calculation of sizes and evaporation of the droplets are results for which I was very involved, and which may be useful for the design and interpretation of the diagnostics in the upcoming target experiments. Also, our research is relevant for the problem of the debris in ICF target chambers in general. It is crucial to know if condensed nanoparticles can form after the explosion, because they could be a serious problem, for example for the optics in a laser configuration.

The results on the third project will also be presented, probably by me, as a poster at the conference of the DIvision of Plasmas Physics of the American physical Society in october 2006 in Philadelphia. 


\section{Appendix 1: Maxwell's image solution for plane sheet of current}

In his classic treatise [8], Maxwell has given a solution for the time-dependent field created by the eddy currents in an infinite thin conductor plate submitted to a varying field.

As explained in section 1.3, the impulsive response is given by an image source, symmetrical with respect to the plate, recessing at the constant speed $v=\frac{2 \sigma}{\mu}$, where $\sigma$ is the surface resistivity of the plate and $\mu$ is its magnetic permeability.

To implement this solution numerically, the method to follow was to make a convolution of the Green function and the current pulse, namely, to integrate the contributions of all the eddy currents generated before the measurement is done, which is at beamtime, when the drive current is at its peak value.

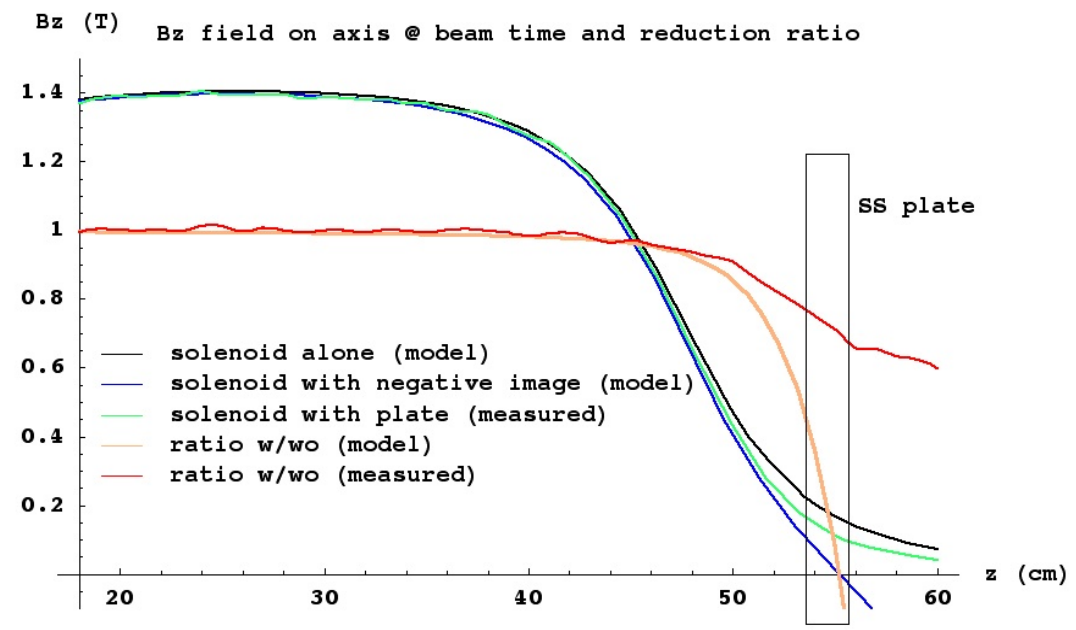

Figure 3.6: Implementation in Mathematica

Figure 6 shows the result of an implementation I did in Mathematica, with only $\mathrm{n}=30$ timesteps, but it was largely enough for the convergence. One sees that the reduction calculated with this model (orange) and the measured one (red) are in fairly good agreement far from the plate (say, from $\mathrm{z}=40 \mathrm{~cm}$ to $\mathrm{z}=48 \mathrm{~cm}$ ), but closer from the plate they start diverging significantly. This is, of course, due to the approximations that were made, in particular the assumption of no hole. 


\section{Appendix 2: Estimation of the relevant thermodynamic functions for the liquid/vapor aluminum flow}

Related to the binding properties: surface tension and latent heat Surface tension and latent heat are two functions directly related to the bonds between atoms in condensed matter.

To estimate the surface tension of liquid aluminum (against its vapor in thermal equilibrum), I first found some experimental data in [20]. These data were old, and there were only three points from $933 \mathrm{~K}$ (melting point) to $1223 \mathrm{~K}$. I looked for more up-to-date values, and I found a recent paper [21], displaying data up to $2000 \mathrm{~K}$, but those measurements had been done with very pure aluminum, with nearly no oxide, and the values of $\sigma$ obtained were significantly bigger than the usual ones. I considered that they were not appropriate and decided not to use them.
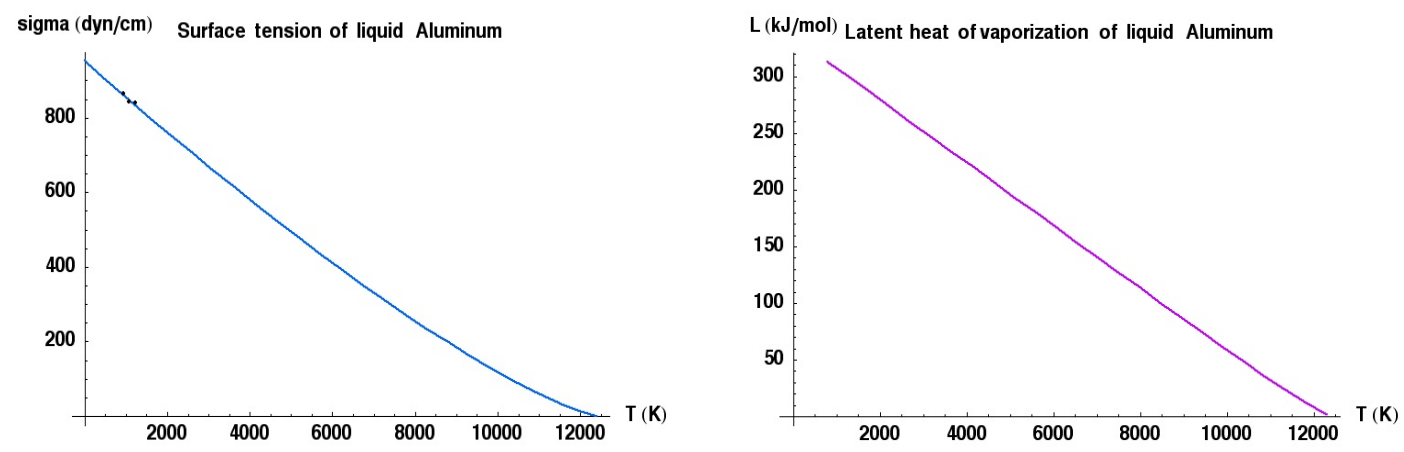

Figure 3.7: Surface tension and latent heat of liquid aluminum

Figure 7a shows a plot of $\sigma(T)$, with the three experimental points already mentioned. To determine the rest of the curve, I used the information that $\sigma$ has to vanish at the critical point, because interfaces do not exist anymore beyond there, and also the law of corresponding states, which says that all substances obeying a Van der Waals EOS (which we supposed to be not too bad an approximation for aluminum in and around the 2-phase region) can be entirely described with the same equations, if one rescales the thermodynamic variables with the critical volume $V_{c}$ and the critical temperature $T_{c}$. I found in [22] that the empirical formula 
$\sigma(T)=\sigma_{0}\left[1-\frac{T}{T_{c}}\right]^{1+r}$, with $\mathrm{r}=0.27 \pm 0.02$ is valid for many simple liquids. So I just adapted that function with $T_{c}$ taken from the EOS in the DPC code, and the three experimental points mentioned here above.

For the latent heat (which finally was not so much needed for our calculations), I first found some experimental values in [23]. Then I considered that $\mathrm{L}(\mathrm{T})$ also has to vanish at the critical point, and for its functional dependence on $\mathrm{T}$, that it is proportional to the energy of the bonds between atoms, so it should be proportional to $\sigma$ too. But, as $\mathrm{L}(\mathrm{T})$ is an energy per unit mass, and $\sigma$ per unit surface, one should write $L(T) \sim \sigma \rho^{-\frac{2}{3}}$, which is computable because $\rho_{\text {liq }}(T)$ is known from the phase diagram of the DPC code.

Figure $7 \mathrm{~b}$ shows a plot of a function proportional to $\sigma \rho^{-\frac{2}{3}}$ that was adjusted to match the experimental values of $\mathrm{L}(\mathrm{T})$. It looks totally linear from 0 to $T_{c}$ ! After some reflexion, we found that this result was very consistent with a simple model for $\mathrm{L}(\mathrm{T})$. Assuming that the liquid phase is a well of potential of a certain fixed depth $L_{0}$, the latent heat can be expressed as the difference $L_{0}-3 k_{B} T$, because it is the energy that is needed for one atom of average energy $3 k_{B} T$ to escape from the well. If the depth of the well is $L_{0}$ independently from $\mathrm{T}$, which is a reasonable assumption on first order, then $\mathrm{L}(\mathrm{T})$ is linear, with slope $-3 k_{B}$, which is found to be well verified in Fig. $7 \mathrm{~b}$ (within $5 \%$ error).

Related to transport properties: viscosity The viscosity was quite difficult to estimate, because theories for the viscosity of liquids are not simple at all. For the gas state, if not too dense, we claim that a kinetic gas model should give correct estimates. The formula used is: $\mu=\frac{m v}{3 \sqrt{2} \sigma_{0}}$ (it does not depend on the density of the gas, only on its thermal velocity).

I also found some experimental values for the viscosity of liquid aluminum [24].

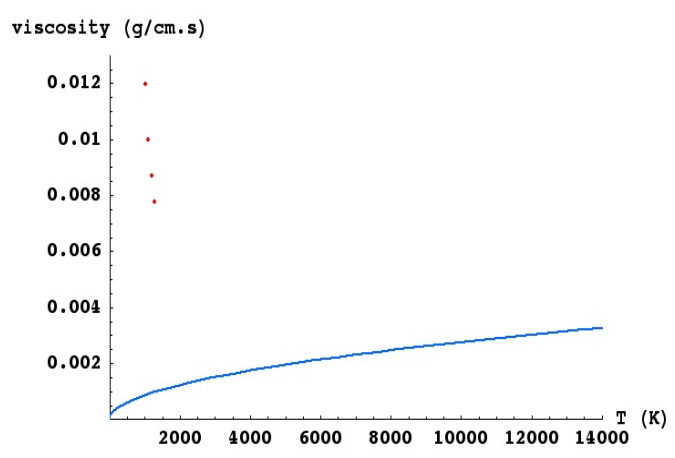

Figure 3.8: Viscosity of liquid aluminum

Figure 8 shows the experimental values for the liquid (red dots) and the model gas viscosity from the kinetic theory (blue line). This graph is satisfactory because it is expected that the viscosity of the liquid is higher than the viscosity of the gas, but decreases with temperature, 
whereas the viscosity of the gas increases ${ }^{7}$.

Of course, both viscosities must become the same at the critical point. All this information is enough to choose values that are not too arbitrary for our estimates.

\footnotetext{
${ }^{7}$ See for example Hildebrand's theory for the viscosity of fluids in [25] or [26]. Hildebrand has shown (with many experimental data) that the fluidity $\Phi=\frac{1}{\mu}$ increases linearly with the volume of the liquid, whereas for gases the fluidity is smaller for bigger volumes. Hildebrand also states that the viscosity of a liquid is very little dependent on the temperature. So, if one considers the dilatation a liquid with temperature, one can understand that the viscosity of a liquid decreases with temperature: as $\mathrm{T}$ goes up, $\mathrm{V}$ goes up to, then the fluidity goes up and the viscosity goes down. The more random motion the particles have, the less able they are to communicate momentum in an organized way.
} 


\section{Acknowledgements}

I would like to thank in first place Peter, who was my advisor for nearly six month, and who was particularly dedicate in helping me build the structure of my work all along my stay at LBNL. He always took time to talk with me and helped me with many problems, scientific or not.

I would like to thank also my two other advisors, Bill and John, because it was great to work with them. Thanks to Bill particularly for initiating me to WARP, and thanks to John for introducing me in the WDM world! A particular thanks to John for the work we did together, because it was really an exciting adventure.

I want to thank also Christine Celata, who made it possible for me to come to Berkeley, and to Grant Logan, head of the HIF group, who welcomed me very gently.

I would like to thank all the other members of the HIF group wit whom I worked: Dick More, David Baca, Prabir Roy, Andy Faltens, Matthaeus Leitner, David Grote, Jean-Luc Vay.

Josh Coleman deserves to be in the previous list, but I prefer thank him, Sven, Christian and Sam for being great student-mates.

I also want to express my recognition to the other members of the group: Frank, Alex, Enrique, Steve, Simon, Shmuel.

A special thanks to Lynn, Wes and Sharon for their help, presence an company,

And, finally, I would like to thank all the persons in Berkeley that made my stay so enjoyable. 


\section{Bibliography}

[1] Tutorial on heavy-ion fusion energy. http://hif.lbl.gov/tutorial/tutorial.html.

[2] P. F. Peterson. Design methods for thick-liquid protection of inertial fusion chambers.

[3] R. M. More. Warm dense matter research. In Proceedings of the Workshop on Accelerator Driven Warm Dense Matter Physics, Pleasanton, California (http:// hifweb.lbl.gov/ public/ AcceleratorWDM/proceedings/ 1.Wednesday.Feb.22/ am09.00.R.More.pdf), february 2006.

[4] P. K. Roy et al. Drift compression of an intense neutralized ion beam. Phys. Rev. Lett., 95:234801, 2005.

[5] E. Henestroza et al. Final focus system for high intensity beams. Phys. Rev. Special Topics - Accelerators and Beams, 8:052801, 2005.

[6] P. A. Seidl et al. Plans for longitudinal and transverse neutralized beam compression experiments and initial results from solenoid transport. In Proceedings of the HIF 06 Symposium, Saint-Malo, France, july 2006. report LBNL-59844.

[7] M. Reiser. Theory and design of charged particle beams. 1994.

[8] J. C. Maxwell. A Treatise on Electricity and Magnetism (part IV: electromagnetism, chapter XII: current-sheets). 1873.

[9] W. R. Smythe. Static and Dynamic Eletricity. 1950.

[10] J.-L. Vay et al. Self-consistent imulations of heavy-ion beams interacting with electronclouds. In Proceedings of the HIF 06 Symposium, Saint-Malo, France, july 2006. Report LBNL-59799.

[11] W. M. Sharp et al. Simulating electron clouds in high-current ion accelerators with solenoid focusing. In Proceedings of the HIF 06 Symposium, Saint-Malo, France, july 2006.

[12] S. Eliezer, A. Ghatak, and H. Hora. Fundamentals of equation of state. 2002.

[13] J .J. Barnard, J. Armijo, R. M. More, and al. Theory and simulation of warm dense matter targets. In Proceedings of the HIF 06 Symposium, Saint-Malo, France, july 2006. Report LBNL-59851.

[14] L. R. Grisham. Moderate energy ions for high energy density physics experiments. Physics of plasmas, 11 (12):5727, 2004. 
[15] R. M. More, H. Yoneda, and H. Morikami. Short-pulse lasers and electron dynamics in warm dense matter. Journal of Quantitative Spectroscopy and Radiative Transfer, 99:409424, 2006.

[16] L. D. Landau and E. M. Lifshitz. Fluid mechanics (2nd edition), Course of theoretical physics, volume 6, chapter X: one-dimensional gas flow. 1987.

[17] J. M. Rallison. The deformation of small viscous drops and bubbles in shear flows. Annual Review of Fluid Mechanics, 16:45-66, 1984.

[18] H. A. Stone. Dynamics of drop deformation and break-up in viscous fluids. Annual Review of Fluid Mechanics, 26:65-102, 1994.

[19] M. Pilch and C. A. Erdman. Use of breakup time data and velocity history to predict the maximum size of stable fragments for acceleration-induced breakup of a liquid drop. International Journal of Multiphase Flow, 13-6:741-757, 1987.

[20] CRC Handbook of Chemistry and Physics. 1964.

[21] V. Sarou-Kanian, F. Millot, and J. C. Rifflet. Surface tension and density of oxygen-free liquid aluminum at high temperature. International journal of Thermophysics, 24 (1), 2003.

[22] F. P. Buff and R. A. Lovett. Simple dense fluids (chapter 2: The surface tension of simple fluids). 1968.

[23] CRC Handbook of Chemistry and Physics, 86th edition. 2005-2006.

[24] M. J. Assael et al. Reference data for the density and viscosity of liquid aluminum and liquid iron. J. Phys. Chem. Ref. Data, 35 (1):285, 2006.

[25] J. H. Hildebrand. Fluidity: a general theory. Proc. Nat. Acad. Sci., 69:3428-3431, 1972.

[26] J. H. Hildebrand and R. H. Lamoreaux. Viscosity along continuous paths between liquid and gas. Physica, 74:416-422, 1974. 